\title{
Supporting Real-Time Data Transmissions in Cognitive Radio Networks Using Queue Shifting Mechanism
}

\author{
B. Seetha Ramanjaneyulu, VFSTR University, India \\ K. Annapurna, VFSTR University, India
}

\begin{abstract}
As cognitive radio networks are conceptualized to make use of the opportunistic spectrum access, the users of these networks may face problems in satisfying their quality of service (QoS) requirements. Some services of users like real-time audio and video which cannot tolerate inter-packet delays will be affected more due to this. The problem occurs due to the non-availability of channels to these applications at some instants. This problem can be addressed if the available channels are judiciously distributed among the competing users. One such mechanism that dynamically allocates the competing users to multiple queues, and shifting the users to higher-level queues as the time elapses is introduced in this work. This is found to help the users of cognitive radio networks to communicate reasonably well even when fewer channels are available for opportunistic use. Results are indicated in terms of blocking probabilities of real-time data. Markov chain-based analysis and discrete event simulation studies are carried out.
\end{abstract}

\section{KEYWORDS}

Cognitive Radio, Markov Chains, Opportunistic Spectrum Access, Primary Users, QoE, QoS, Queue Shifting, Real-Time Transmission, Secondary Users

\section{INTRODUCTION}

Under-utilization of spectrum bandwidths allocated to various licensed services, has given rise to the concept of Cognitive Radio Networks (CRN). It is based on the proposal of using those underunutilized bandwidths by non-licensed users, through opportunistic spectrum access (Mitola J., 2009). The main advantage of the cognitive radio networks is that they make better use of those bandwidths. If this mechanism is adopted in those frequency ranges in which many of the mobile and cellular communication systems are operating, it will be extremely helpful, because those bands are crowded heavily and hardly any bandwidth is available at government agencies to allot it to new services. In fact, there are some underutilized spectrum frequencies in these frequency ranges, which were originally licensed to terrestrial TV operations, and various applications of military and navigation (Young-June Choi, Shin K.G., 2011). So, cognitive radio networks are vying for better use of these 
frequencies. In the terminology of cognitive radio networks, the licensed users are called 'Primary Users' (PU) and the opportunistic cognitive radio users are called 'Secondary Users' (SU).

As sharing of available spectrum among Secondary Users is the most important aspect of cognitive radio networks, several proposals are made by researchers, for this sharing. Based on the way SUs avail the spectrum opportunity, three types of spectrum sharing paradigms are available (Goldsmith, A., Jafar, S.A., Maric, I. \& Srinivasa, S., 2009). They are:

1. Underlay Methods: Here PUs and SUs coexist together such that SUs will use less power and hence do not disturb the PUs' communications.

2. Overlay Methods: Here SUs will make use of spectrum holes with the help of messages and code books shared by PUs. In return, SUs need to help in relaying the transmissions of PUs.

3. Interweave Methods: In this paradigm, SUs should continuously sense the spectrum for finding and exploiting the spectrum holes.

Of the above methods, overlay type of spectrum sharing is simple to implement, and hence the preferred one, in many contexts. While sharing the spectrum, allocation of channels to individual users can be made either through central administration or through distributed mechanisms. In the case of central decision making methods, one central system decides all the channel allocations in that region by collecting the data from all the cognitive radios of that region about their measurements and as well as the channel requirements. Measurements here refer to the sensing of spectrum activity by that cognitive radio. Energy detection is the commonly used method of spectrum sensing (Won-Yeol Lee; Akyildiz, I.F., 2008). In the case of distributed decision making methods, each cognitive radio takes the decision on its own by checking its measurements for all channels in its surroundings and then selecting a free channel for its communication. This kind of decision may be required in the cases where the cognitive radios operate in an environment where proper structure of the network doesn't exist. In the proposed work of this paper, centralized channel allocation is considered.

All the users of cognitive radio networks, especially the ones that use overlay methods of spectrum sharing, have to follow the basic principle of vacating the occupied channels whenever the licensed users of the spectrum want to use those frequencies. In such instants, the vacated SU needs to search for another vacant channel immediately to continue its communication (Elias Z. Tragos, Sherali Zeadally, Alexandros G. Fragkiadakis \& Vasilios A. Siris, 2013). If it fails to find another vacant channel, then it needs to quit the system, which is called as dropping the transmission that will lead to degradation of quality of service (QoS) to SUs. This impact may not be same for all the users. For some users who are engaged in non time-sensitive communication like the e-mail transfer, it may not appear to be that severe. But for some users who are engaged in time-sensitive data transfers like the real-time audio, the impact is clearly evident. In such cases, if the cognitive radio transmission system is designed in such a way that it takes into consideration the time-sensitivities of user data, it may be able to fulfill the user requirements in a better way. One such proposal is introduced in this work. It is based on queuing systems. Multiple queues are considered, into which user data packets are queued in, based on their time-sensitive requirements. Of all these queues, the packets that travel in the first queue only are passed to the transmission system. That means, the packets that are put to different other queues need to shift from the lower-queues to the upper-queues, and finally to the first queue, as the time elapses. Time sensitiveness of the data packet and the time it already spent in the queues will decide its movement to the upper-level queues, and finally to the first queue from where it is transmitted. It can be considered as M/M/1 type of system, because single service of transmission system is considered. It can be extended to multiple number of services also, with ease. The proposed system is analyzed using Markov chain models and simulated with discrete event simulations. The proposed system is based on the concept of distributing the instant channel bandwidths to the needed users, as per their packet transmission urgencies. 
Rest of the paper is organized as follows. Section-2 of it discusses about various spectrum sharing methods proposed in literature for cognitive radio networks. It discusses some of the proposals that dealt with real-time transmissions of users and the various queuing methods proposed for cognitive radio networks. Section-3 discusses the details of the proposed method. Models of the system that include the proposed method with possible scenarios and their analysis are presented in Section-4. Section-5 presents the simulation studies carried out and their results. Section- 6 concludes the paper.

\section{SPECTRUM SHARING TECHNIQUES}

Sharing of the vacant spectrum among secondary users is one of the prime aspects of Cognitive Radio Networks. The challenges of spectrum sharing in CRNs are numerous because of the continuous change of available bandwidths, inaccuracies in detecting the spectrum holes, and the like. Assumptions like SUs knowing the power and location of PUs so as to calculate the interference also lead to some other problems (Akyildiz, I. F., Lee, W. Y., Vuran, M. C. \& Mohanty, S., 2008). After identifying the vacant channels, the usual approach of spectrum sharing is to treat all the users the same way and try to optimize the allocation. Another approach is to take into account the user requirements and thereby offer prioritized allocation of channels to some users. Most of these later methods are considered as real-time supporting methods. Some non-real-time and real-time supporting methods of spectrum sharing are discussed here.

One popular paradigm of spectrum sharing is cooperative vs. non-cooperative sharing. In cooperative spectrum sharing schemes, all the radios in the region cooperate with each other by exchanging their channel measurement and channel requirement information. In fact, the central decision mechanism considered for this paper also needs this kind of cooperation. In (Jayaweera, S. K., Vazquez-Vilar, G. \& Mosquera, C., 2010), the authors expressed the cooperation between PUs and SUs as PUs will offer bandwidth or time slots to SUs when PUs' weak transmissions are relayed by SUs' powerful transmitters. As the PUs' transmissions get finished fast with the help of SUs, the leftover time can be offered to secondary users without a need of really sensing the spectrum or without the dilemma of vacating it when the PU returns back. However, it happens only when PUs need SUs' services.

Another type of spectrum sharing is based on game theory, where the users will define the utility function and play their games to maximize their utility functions. The important proposals in literature on game theory based mechanisms are the works proposed in (Yu Peng Xie, Xuezhi Tan, Yu Tao Liu, Lin Ma, Hai Yan Wu., 2011; Beibei Wang, Yongle Wu, K. J. Ray Liu, 2010; Niyato, D.\& Hossain, E., 2007):

In (Liang, Z., Feng, S., Zhao, D. \& X. S. Shen., 2011), the authors have proposed to support real-time transmissions, by allowing the prioritized users to use the available channels whenever they want to transmit the data. When such prioritized transmissions are not there, then only the normal users would get the channels.

In (Kunert, K., Jonsson, M. \& Bilstrup, U., 2012), the authors have proposed the channel allocations to users of different priorities, based on assigning different inter frame spacing (IFS) intervals to different users. That means high-priority users will be assigned with lesser values of IFS compared to low-priority users. It is similar to the IFS mechanism used in IEEE802.11 MAC algorithm, where three different IFS values namely DIFS, PIFS and SIFS were proposed to be used with Adhoc WLAN connections, infrastructure WLANs and for the transmission of acknowledgement packets in either type of WLANs (IEEE Std 802.11, 2016).

Another method that supports prioritized transmissions is introduced in (Yang, W. \& Zu, Y., 2016). To maintain QoS of SUs, the authors used M-LDWF (Maximum Largest Weighted Delay First) algorithm, in which the users who have waited for more time will be selected each time from the queue. In addition to this, they have classified SUs into two types as latency sensitive and latency 
insensitive. For each type of SUs they have used two queues, one for newly arriving SUs and the other for handoff SUs.

In (El Helou, M., Ibrahim, M., Lahoud, S., Khawam, K., Mezher, D.\& Cousin, B., 2015), the authors addressed the problem of selfish users, because of whom, the system performance degrades. The main concept here is, if secondary users are provided with network parameters like QoS parameters and cost, SUs combine them with needs and preferences and will take better decisions. As supplying the network parameters is not an easy task, they introduced reinforcement learning algorithm to obtain the network parameters. Semi-Markov Decision Process (SMDP) was used to derive the network information, which meets the objectives of both the operator and SU. In (Annapurna, K. \& Seetha Ramanjaneyulu, B., 2019), QoS to SUs is offered through an auction mechanism in which bid values are predefined and priority of SUs is considered in terms of their willingness to pay.

In the above discussion, channel allocation does not necessarily mean a dedicated carrier frequency along with a quantity of bandwidth allocated to the user. Most of the times, the allocations can be in the form of time slots during which the users will be permitted to use the designated frequencies. To carry out such allocations, queue based mechanisms are helpful. Some of the important works that dealt with queuing-theory based proposals for channel allocations in cognitive radio networks are presented here.

In (Balapuwaduge I.A.M., Jiao L., Pla V and Li F.Y, 2014), the authors proposed a system with two queues, one for the real-time and the other for elastic traffic. Then channel access opportunities are distributed between the two queues, with more opportunities provided to real-time supporting queue. For the queue that deals with elastic traffic, buffering option is proposed through which the packets that are dropped at a given instant due to the non-availability of channel may be put back in the queue for transmission. In (Suganthi N \& Meenakshi S, 2018), the data is considered to have upto five different priority classes. Two queues are proposed onto which these five classes of data packets are distributed. Usually high priority devices get the access whenever competition arises. However, a classification mechanism that is based on Fuzzy inference may decide the channel allocation to low priority devices also, based on the combined RRP (round robin priority) scheduling algorithm proposed in the work. In (F. Wang, J. Huang \& Y. Zhao., 2012), the authors have proposed a threshold based optimal admission control policy combined with largest delay first algorithm, for channel allocation in cognitive radio overlay networks. It was formulated as Markov decision process problem and was meant for supporting delay sensitive communications of SUs.

In (Homayounzadeh A \& Mahdavi M, 2017), the authors have analyzed the quality of service for real time secondary users of overlay cognitive radio network, using Markov chains with the consideration of sensing errors. Time-sensitive data like that of cellular communication system is considered. The authors have proposed a packet scheduling algorithm where Channels are classified based on their SNR values and the scheduling algorithm is meant to assign suitable channels to timesensitive data. In (H. Shiang and M.\& van der Schaar, 2008), the authors proposed a virtual queue interface for secondary users of cognitive radio networks, who want to transmit the delay-sensitive multimedia data. An algorithm that exploits the expected delay and dynamically adopts channel selection strategies to maximize users' utility functions is proposed.

In (Feng $S$ \& Zhao D, 2010), the authors proposed cognitive radio based channel access to wireless sensor networks. Data is divided into two types namely the real-time and best effort (BE). Two resource allocation policies are proposed in order to provide high priority to real time secondary users. They are absolute priority and reservation based mechanisms. In absolute priority, it first serves all the real time data and if channels are still available, it then serves the BE traffic. In reservation based policy real time data will be served during reservation timeslots and this information will be made available to all sensors so that the sensors which are having only BE data can enter sleep mode during that time.

In most of the above works, two queues are proposed, one for the real-time and the other for nonreal-time traffic. In the works like that of (Wang F., Huang J \& Zhao Y., 2012), 'largest delay first' 
policy is used. In our proposed work, a combined approach is adopted, where delay after entering the queue is coupled with the time-sensitiveness of the data, to arrive at the channel allocation decision. To implement it appropriately, queue shifting mechanism is proposed, which is discussed in the next section.

\section{PROPOSED QUEUE SHIFTING TECHNIQUE}

The proposed method of queue-shifting considers the system to have multiple queues, for example four in this discussion. Each of these queues is assigned with a value in terms of time units. Likewise, the data packets that are delivered to the network by various secondary users include a 'Tolerable Interval' value in them. This value is nothing but the maximum possible time delay the packet can wait in the queues. So the transmission system should take care to process the packets before their time-delay durations expire. For this to be tracked properly, timers are associated with interval field. Initially, after the user packets arrive at the system, the system checks the interval durations of each packet and then puts them to appropriate queues, based on these interval values. The packets that jump to the first queue are connected to transmitter line and hence all these packets that enter this queue get the opportunity of being transmitted one after the other, in the order in which they entered the queue. Now, as the time elapses, the timer values of the packets that are put to lower queues get decreased gradually, making the packets to move to the upper queues whenever their residual intervals fall below the threshold value of the upper-rung queue. If the buffer of that upper queue is full, the packet will continue in the lower-rung queue though its timer interval will be decreasing as usual. On the other side, if the buffer of upper-queue is empty, then the packet of lower-rung queue will be brought to that upper-queue, even before its timer value reaches the threshold value of the queue. This mechanism is to ensure that the bandwidths are not wasted.

Whether all the packets that entered the system will get the transmission opportunity or not is based on the processing capacity of the transmission system. That means, if sufficient number of vacant channels are available, then it will be able to process all the packets that entered the system. If sufficient number of vacant channels are not available, some packets may not get the chance of moving to upper-rung queues and hence lose the transmission opportunity. As evident from the discussion, this happens to the packets whose time interval values start at very high values. So, high-priority data should be assigned with lower interval values and low-priority data should be assigned with higher interval values. So the appropriate term 'Tolerable Duration' is given to this parameter.

\section{SYSTEM MODEL}

Markov chain based analysis is considered. The PU occupancy information can be modelled as Markov chain as shown in Fig-1 (Alshamrani, A., Shen, X. S. \&Xie, L. L., 2011). The primary channel occupancy can be written as:

$$
\delta_{i}=\frac{\beta_{i}}{\alpha_{i}+\beta_{i}} \text { for } \mathrm{i}=1,2,3, \ldots \mathrm{N}
$$

where, $\alpha_{i}$ represents the channel transition probability from occupied to idle state, and $\beta_{i}$ represents the probability of channel transition from idle to occupied state

The system supports pre-emption, in which a low priority SU needs to vacate the channel when a high priority SU needs it. 


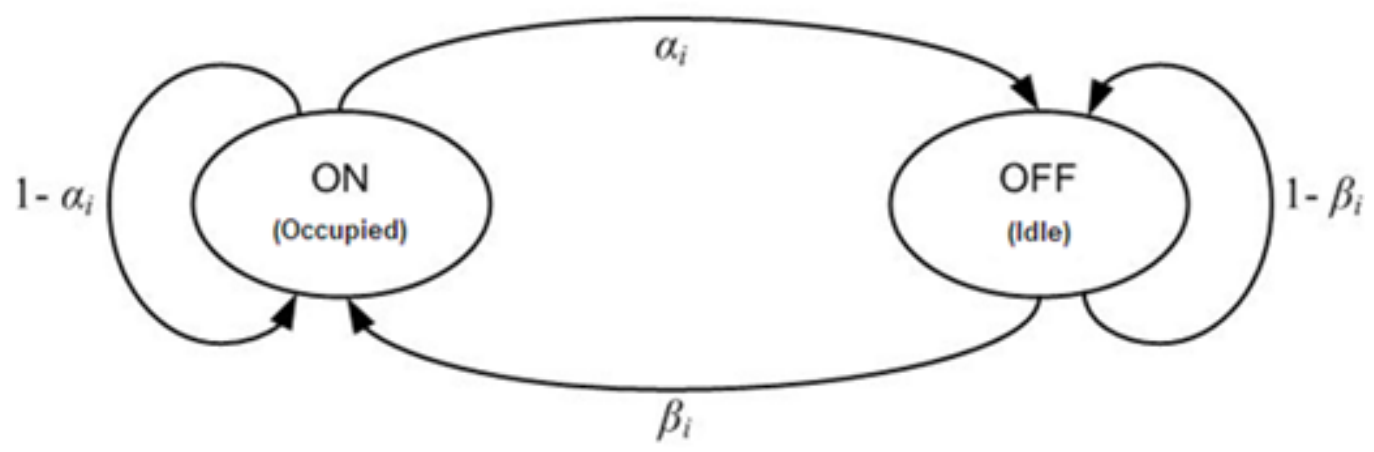

\section{State Space Model to Derive Blocking Probability}

In (Peter J. Smith, Abdulla Firag, Pawel A. Dmochowski, and MansoorShafi, 2012), blocking probabilities are derived for PUs and SUs for three cases namely, Secondary User Cleared (SUC), Secondary User Equality (SUE) and Secondary User Partially Cleared (SUPC). Here, SUC means when a PU arrives if no free channels are available, then the SU that is in service should be dropped, which is nothing but preemption. In this scenario, no sensing errors are considered. In SUE, both SUs and PUs are treated equally - that means no preemption occurs and so it is equal to the case of imperfect sensing and SU will continue its transmission due to misdetection. In SUPC, the SU will drop partially when PU arrives. That is, sensing is neither perfect nor imperfect.

In the proposed work, SUs are divided into four priority classes SU1, SU2, SU3 and SU4. The priority decreases from SU1 through SU4.

Let there are N channels in the system where PUs and SUs follow Poisson distribution for arrivals and exponential distributions for service times. Let the arrival rate of PUs is $\lambda_{\mathrm{p}}$, and the arrival rates of SU1 through SU4 are $\lambda_{\mathrm{s} 1}, \lambda_{\mathrm{s} 2}, \lambda_{\mathrm{s} 3}$ and $\lambda_{\mathrm{s} 4}$. Let the service rates of PUs is $\mu_{\mathrm{p}}$, and the service rates of SU1 through SU4 are $\mu_{\mathrm{s} 1}, \mu_{\mathrm{s} 2}, \mu_{\mathrm{s} 3}$ and $\mu_{\mathrm{s} 4}$. Let the state of the system be represented by $\left(\mathrm{n}_{\mathrm{p}}, \mathrm{n}_{\mathrm{s} 1}, \mathrm{n}_{\mathrm{s} 2}\right.$, $\mathrm{n}_{\mathrm{s} 3}, \mathrm{n}_{\mathrm{s} 4}$ ), where:

$\mathrm{n}_{\mathrm{p}}=$ Number of in-service PUs

$\mathrm{n}_{\mathrm{s} 1}^{\mathrm{p}}=$ Number of in-service SU1s

$\mathrm{n}_{\mathrm{s} 2}=$ Number of in-service SU2s

$\mathrm{n}_{\mathrm{s} 3}=$ Number of in-service SU3s

$\mathrm{n}_{\mathrm{s} 4}=$ Number of in- service SU4s

At every discrete interval of time, based on the arrivals or departures of PUs and SUs, states may change. Here three cases are considered for deriving blocking probability by considering $\mathrm{N}=1$.

Case-1: System with four prioritized SUs, imperfect sensing, without preemption and without queue shifting.

Markov model for case-1 is shown in Figure 2. Writing the flow balance equations at each state results in:

$\mathrm{P}_{0,0,0,0,0}\left(\lambda_{\mathrm{p}}+\lambda_{\mathrm{s} 1}+\lambda_{\mathrm{s} 2}+\lambda_{\mathrm{s} 3}+\lambda_{\mathrm{s} 4}\right)=\mu_{p} p_{1,0,0,0,0}+\mu_{s 1} p_{0,1,0,0,0}+\mu_{s 2} p_{0,0,1,0,0}+\mu_{s 3} p_{0,0,0,1,0}+\mu_{s 4} p_{0,0,0,0,1}$ 


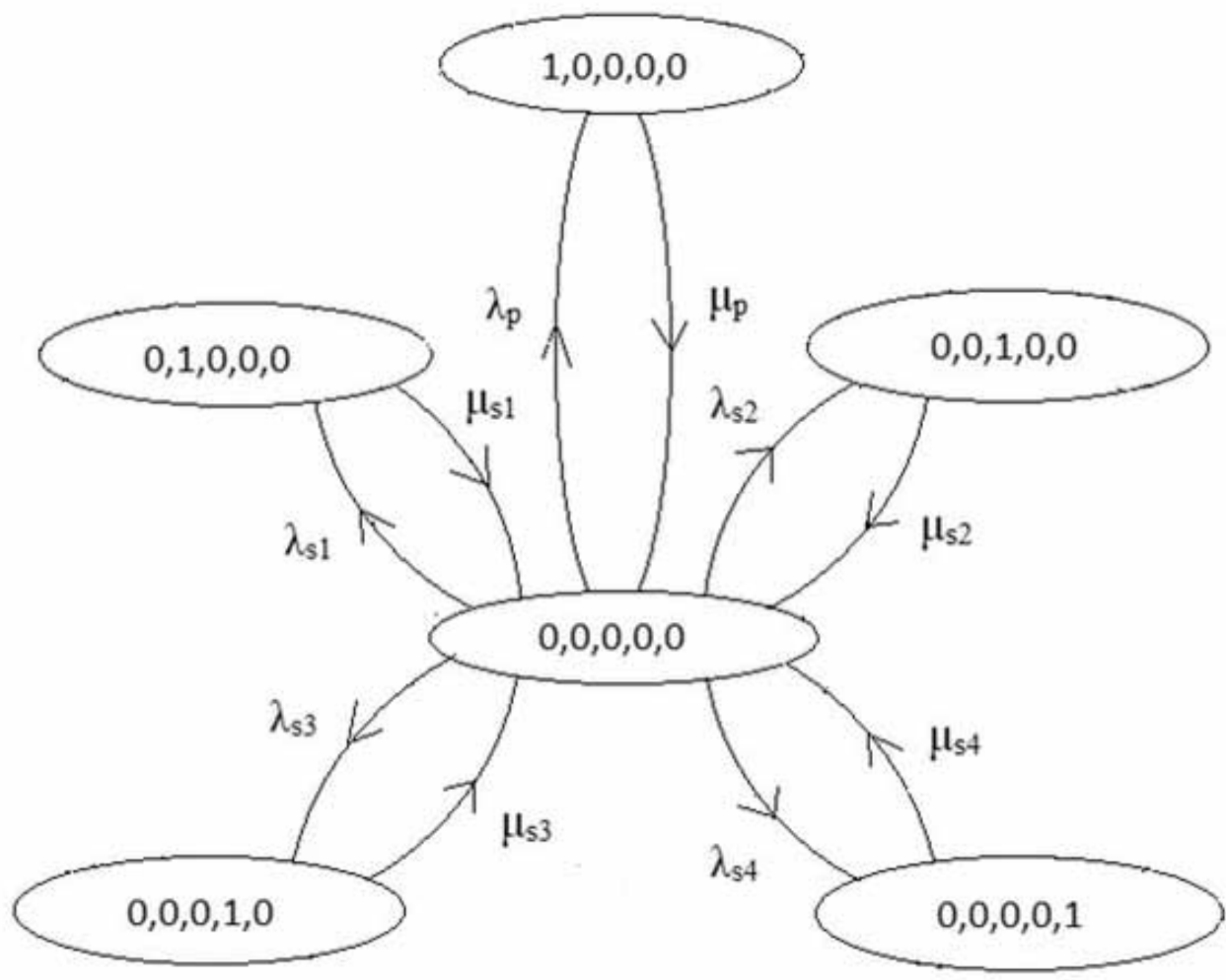

$p_{0,0,0,0,0} \lambda_{p}=p_{1,0,0,0,0} \mu_{p}$

$p_{0,0,0,0,0} \lambda_{s 1}=p_{0,1,0,0,0} \mu_{s 1}$

$p_{0,0,0,0,0} \lambda_{s 2}=p_{0,0,1,0,0} \mu_{s 2}$

$p_{0,0,0,0,0} \lambda_{s 3}=p_{0,0,0,1,0} \mu_{s 3}$

$p_{0,0,0,0,0} \lambda_{s 4}=p_{0,0,0,0,1} \mu_{s 4}$

Sum of all the steady state probabilities is one. So: 


$$
p_{0,0,0,0,0}+p_{1,0,0,0,0}+p_{0,1,0,0,0}+p_{0,0,1,0,0}+p_{0,0,0,1,0}+p_{0,0,0,0,1}=1
$$

By solving equations 2 to 8 , all the state probabilities can be obtained.

Blocking probability of all SU types $=p_{1,0,0,0,0}+p_{0,1,0,0,0}+p_{0,0,1,0,0}+p_{0,0,0,1,0}+p_{0,0,0,0,1}$

That is, if the channel is occupied by any user, the new SU request will be blocked.

Case-2: System with four prioritized SUs, perfect sensing without preemption and without queue shifting.

Markov chain for case-2 is shown in Fig-3. Writing the flow balance equations at each state results in:

$$
\begin{gathered}
\mathrm{P}_{0,0,0,0,0}\left(\lambda_{\mathrm{p}}+\lambda_{\mathrm{s} 1}+\lambda_{\mathrm{s} 2}+\lambda_{\mathrm{s} 3}+\lambda_{\mathrm{s} 4}\right)=\mu_{p} p_{1,0,0,0,0}+\mu_{s 1} p_{0,1,0,0,0}+\mu_{s 2} p_{0,0,1,0,0}+\mu_{s 3} p_{0,0,0,1,0}+\mu_{s 4} p_{0,0,0,0,1} \\
\left(p_{0,0,0,0,1}+p_{0,0,0,1,0}+p_{0,0,1,0,0}+p_{0,1,0,0,0}+p_{0,0,0,0,0}\right) \lambda_{p}=p_{1,0,0,0,0} \mu_{p}
\end{gathered}
$$

\section{Figure 3. Markov Model for case-2}

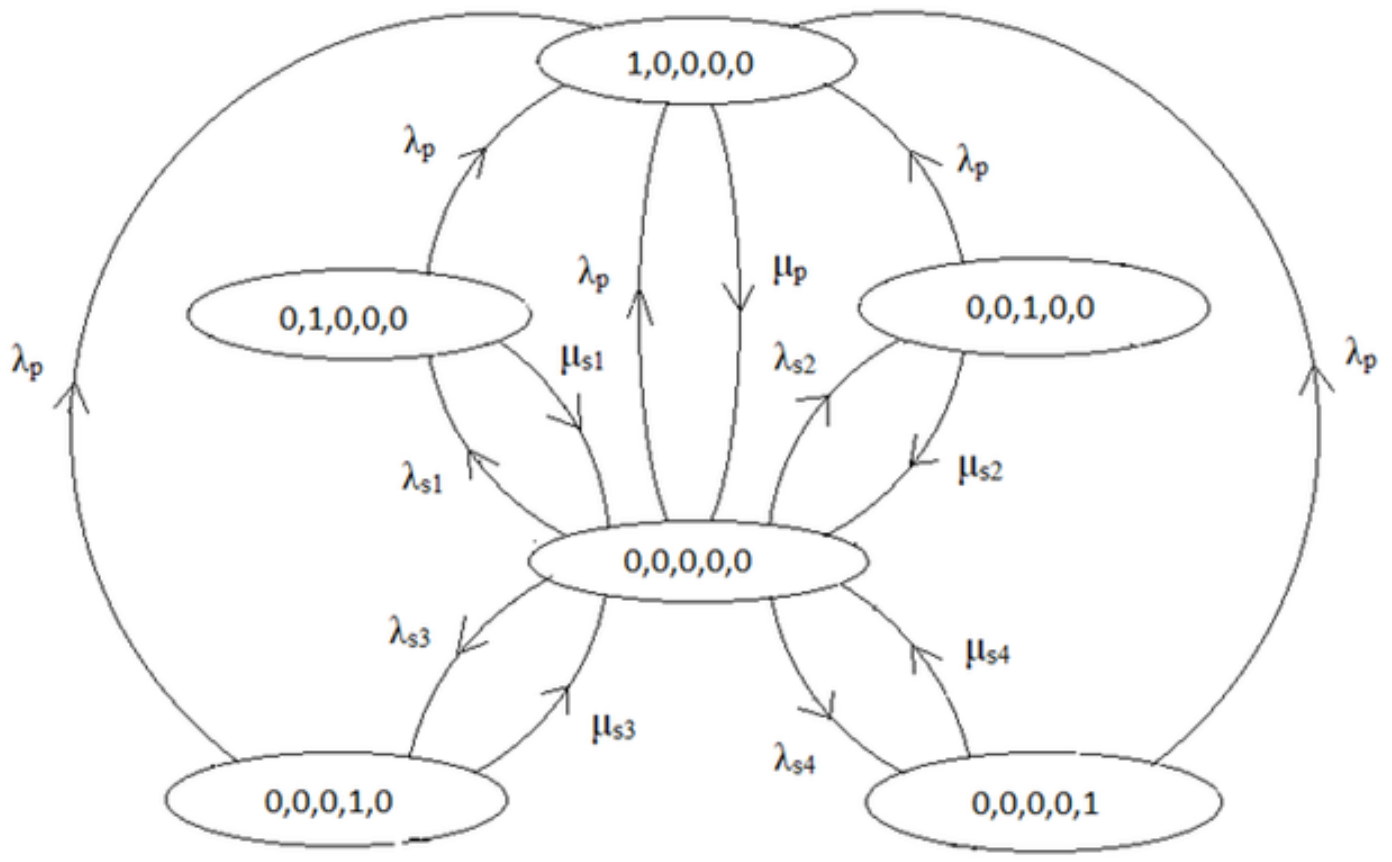




$$
\begin{aligned}
& p_{0,0,0,0,0} \lambda_{s 1}=p_{0,1,0,0,0}\left(\mu_{s 1}+\lambda_{p}\right) \\
& p_{0,0,0,0,0} \lambda_{s 2}=p_{0,0,1,0,0}\left(\mu_{s 2}+\lambda_{p}\right) \\
& p_{0,0,0,0,0} \lambda_{s 3}=p_{0,0,0,1,0}\left(\mu_{s 3}+\lambda_{p}\right) \\
& p_{0,0,0,0,0} \lambda_{s 4}=p_{0,0,0,0,1}\left(\mu_{s 4}+\lambda_{p}\right)
\end{aligned}
$$

Sum of all the steady state probabilities is one. So:

$$
p_{0,0,0,0,0}+p_{1,0,0,0,0}+p_{0,1,0,0,0}+p_{0,0,1,0,0}+p_{0,0,0,1,0}+p_{0,0,0,0,1}=1
$$

By solving Eqns. 10 to 16, all state probabilities can be obtained.

Blocking probability of all SU types $=p_{1,0,0,0,0}+p_{0,1,0,0,0}+p_{0,0,1,0,0}+p_{0,0,0,1,0}+p_{0,0,0,0,1}$

Case-3: System with four prioritized SUs, perfect sensing with preemption and without queue shifting.

Markov chain for case-3 is shown in Figure 4. Writing the flow balance equations at each state results in:

$$
\begin{aligned}
& \mathrm{P}_{0,0,0,0,0}\left(\lambda_{\mathrm{p}}+\lambda_{\mathrm{s} 1}+\lambda_{\mathrm{s} 2}+\lambda_{\mathrm{s} 3}+\lambda_{\mathrm{s} 4}\right)=\mu_{p} p_{1,0,0,0,0}+\mu_{s 1} p_{0,1,0,0,0}+\mu_{s 2} p_{0,0,1,0,0}+\mu_{s 3} p_{0,0,0,1,0}+\mu_{s 4} p_{0,0,0,0,1} \\
& \left(p_{0,0,0,0,1}+p_{0,0,0,1,0}+p_{0,0,1,0,0}+p_{0,1,0,0,0}+p_{0,0,0,0,0}\right) \lambda_{p}=p_{1,0,0,0,0} \mu_{p} \\
& \left(p_{0,0,0,0,0}+p_{0,0,1,0,0}+p_{0,0,0,1,0}+p_{0,0,0,0,1}\right) \lambda_{s 1}=p_{0,1,0,0,0}\left(\mu_{s 1}+\lambda_{p}\right) \\
& \left(p_{0,0,0,0,0}+p_{0,0,0,1,0}+p_{0,0,0,0,1}\right) \lambda_{s 2}=p_{0,0,1,0,0}\left(\mu_{s 2}+\lambda_{p}+\lambda_{s 1}\right)
\end{aligned}
$$




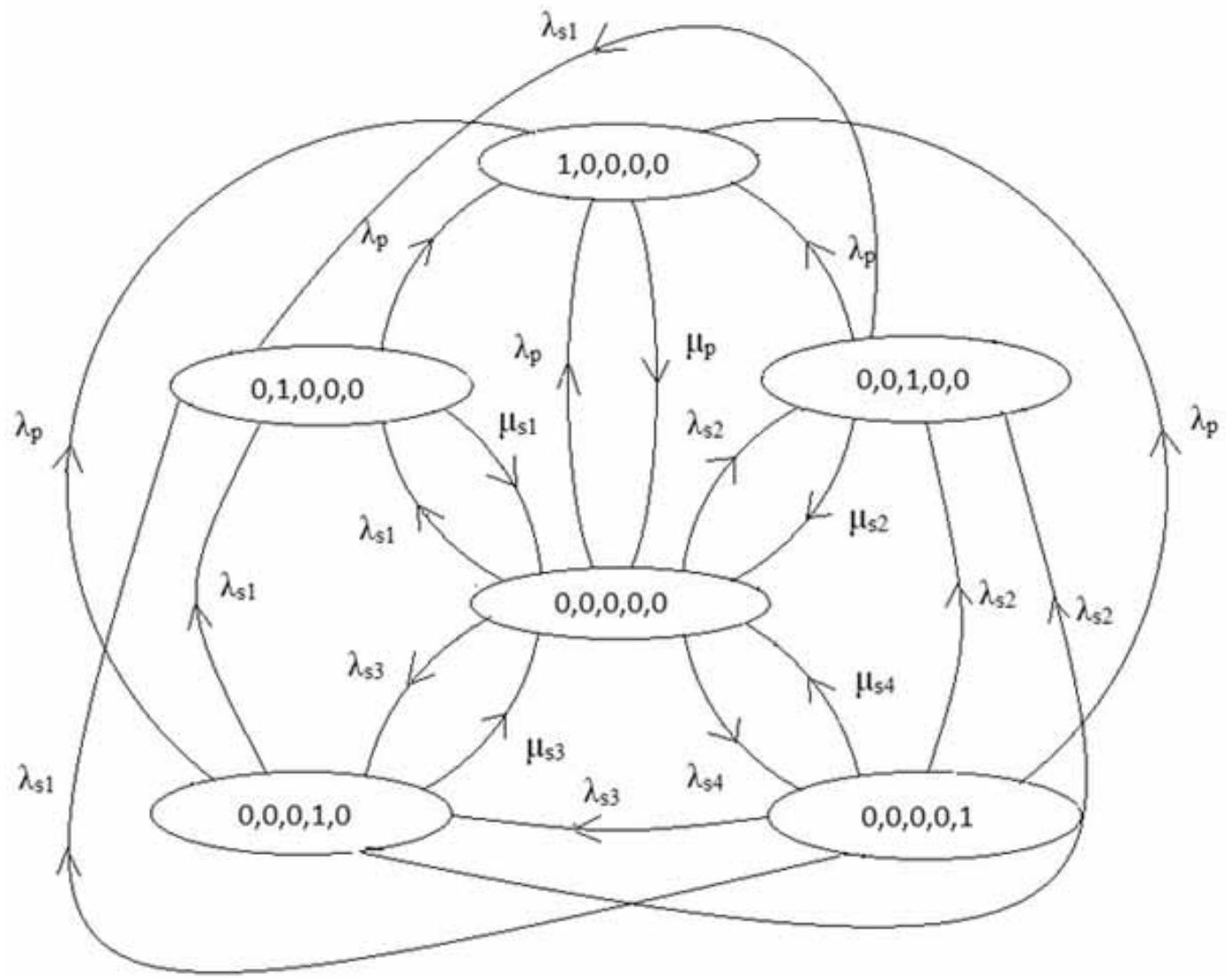

$\left(p_{0,0,0,0,0}+p_{0,0,0,0,1}\right) \lambda_{s 3}=p_{0,0,0,1,0}\left(\mu_{s 3}+\lambda_{p}+\lambda_{s 1}+\lambda_{s 2}\right)$

$p_{0,0,0,0,0} \lambda_{s 4}=p_{0,0,0,0,1}\left(\mu_{s 4}+\lambda_{p}+\lambda_{s 1}+\lambda_{s 2}+\lambda_{s 3}\right)$

As the sum of all steady state probabilities is one:

$p_{0,0,0,0,0}+p_{1,0,0,0,0}+p_{0,1,0,0,0}+p_{0,0,1,0,0}+p_{0,0,0,1,0}+p_{0,0,0,0,1}=1$

By solving 2.18 to 2.24 , one can get all state probabilities.

Blocking probability of SU1s $=p_{1,0,0,0,0}+p_{0,1,0,0,0}$ 
Blocking probability of SU2s $=p_{1,0,0,0,0}+p_{0,1,0,0,0}+p_{0,0,1,0,0}$

Blocking probability of SU3s $=p_{1,0,0,0,0}+p_{0,1,0,0,0}+p_{0,0,1,0,0}+p_{0,0,0,1,0}$

Blocking probability of SU4s $=p_{1,0,0,0,0}+p_{0,1,0,0,0}+p_{0,0,1,0,0}+p_{0,0,0,1,0}+p_{0,0,0,0,1}$

It can be understood from equations 25 to 28 that the blocking probability is increasing when priority is decreasing.

Case-4: System with four prioritized SUs, perfect sensing with preemption and with queue shifting.

Here, when the timer value of the packet decreases, SU packet will be shifted from its present priority level to its next higher priority level, whenever the timer value approaches the threshold value of that upper level queue. So blocking of real-time SUs is further reduced with the introduction of this queue shifting, because the 'Tolerable Interval' values are small here, and hence move to upper level queues quickly.

\section{RESULTS AND DISCUSSION}

Discrete event simulations of the scenarios described above are carried out using Matlab. In the simulated environment, all the devices are able to read all the available data of variables from MATLAB environment. Hence the central entity clearly knows about the availability of free channels and priority requirements of all the devices. However, in the real environment this information will be transmitted on the forward and reverse control channels, in the specified frame formats.

Tables 1 and 2 contains the details of maximum possible values considered for 'Holding Time' and 'Number of Times', while carrying out the simulation,, for the cases of High and Low occupancy of PU and SU are provided. These are given in 'Holding Time' refers to the duration for which the

Table 1. Simulation parameters of PUs

\begin{tabular}{|l|c|c|}
\hline \multicolumn{1}{|c|}{ Parameter } & High PU Occupancy & Low PU Occupancy \\
\hline Maximum number of channels & 10 & 10 \\
\hline Maximum holding time of Pus & 20 minutes & 10 minutes \\
\hline Maximum number of times each PU can reappear & 20 & 10 \\
\hline
\end{tabular}

Table 2. Simulation parameters of SUs

\begin{tabular}{|l|c|c|}
\hline \multicolumn{1}{|c|}{ Parameter } & High SU Demand & Low SU Demand \\
\hline Maximum holding time of SUs & 20 minutes & 10 minutes \\
\hline Maximum number of times each SU is reappearing & 20 & 10 \\
\hline Number of SUs in each type & 20 & 10 \\
\hline
\end{tabular}


device wants to communicate on the channel. 'Number of Times' refers to the count of how many such communication needs arise in a day. 'Random number generator' is used to create a value at the given instant, for these parameters. Table- 3 contains the information about the overall system considered.

In the literature, as discussed in the previous sections, real-time transmission needs are proposed to be met by differentiating the user data as one of the two types, namely the Real-Time (RT) and the Non-Real-Time (NRT). Then the RT data packets coming from users are kept in queue in their order of arriving at the system. Then they are offered with the available channels. After these RT packets are serviced, then opportunities are given to NRT packets. In this type of methods, the time deadlines of RT packets is not considered. That means, if an RT packet whose deadline has approached is there in the queue much behind, and the other RT packets whose deadlines have not approached yet are there ahead in the queue, still the packet in question cannot be brought to front. This case of priority mode is taken and simulated at first. The results are shown in Fig-5. At the first glance, it appears that there is an improvement for the RT packets. But, further analysis of it reveals that the number of RT packets that are delivered within their deadline times are equal to NRT packets. That means, in terms of blocking probabilities, the values are same, which indicates that they are equally blocked like the NRT packets. So, even though RT packets have less blocking probabilities overall, the number of packets that are serviced within their time deadlines are not satisfactory. Simulations are conducted for the case of low SU demand and high PU occupancy. As can be seen from the figure, blocking probabilities get reduced, when more number of channels become available, which is indicated on $\mathrm{x}$-axis.

Results of the proposed method of multiple queues and queue shifting are shown in Figure 6 and Figure 7.

Four queues are considered at the system entry point. That means user packets are put into one of these queues, based on their deadline times. As time passes, residual values of the timer deadlines come down, and hence the shifting of the packets to upper-level queue takes place. Based on their initial time deadlines, the packets can be considered as multiple types, which are equal to the number of queues. If four queues are considered, then four types of packets are considered namely SU1, SU2, SU3 and SU4. To compare the results with RT-NRT methods, SU4 type are considered as NRT and SU1 through SU3 are the RT type. Their actual blocking probabilities and the blocking values after taking the time deadlines also into consideration, are shown in the figure. Finally, the net blocking values, by taking the summation of SU1 through SU3 are given in Fig-7, in comparison with the existing RT-NRT methods. Improvement of upto $20 \%$ maximum is observed. Higher improvements are possible for the case of high SU demand. Simulation results of this scenario are shown in Figure 8 to Figure 10.

Figure 8 is for RT-NRT methods. Figure 9 offers the results of queue shifting method. Finally Fig-10 shows the comparison of the proposed method with the existing RT-NRT methods. Difference

Table 3. Parameters of the overall system

\begin{tabular}{|l|l|}
\hline \multicolumn{1}{|c|}{ Parameter } & \multicolumn{1}{c|}{ Value } \\
\hline $\begin{array}{l}\text { Number of users in each SU category } \\
\text { (i.e., SU1 through SU4) }\end{array}$ & 1 each \\
\hline No of Servers & 1 \\
\hline Transmission Delay (from User to System) & 0 \\
\hline Number of queues considered & 4 \\
\hline Threshold Intervals of the Queues & $\begin{array}{l}\text { Multiples of 10 milli Units of } \\
\text { Time Unit }\end{array}$ \\
\hline Bit Errors / Packet Errors & Nil \\
\hline
\end{tabular}


Figure 5. Blocking Probability of RT and NRT Secondary Users for High PU Occupancy and Low SU Demand

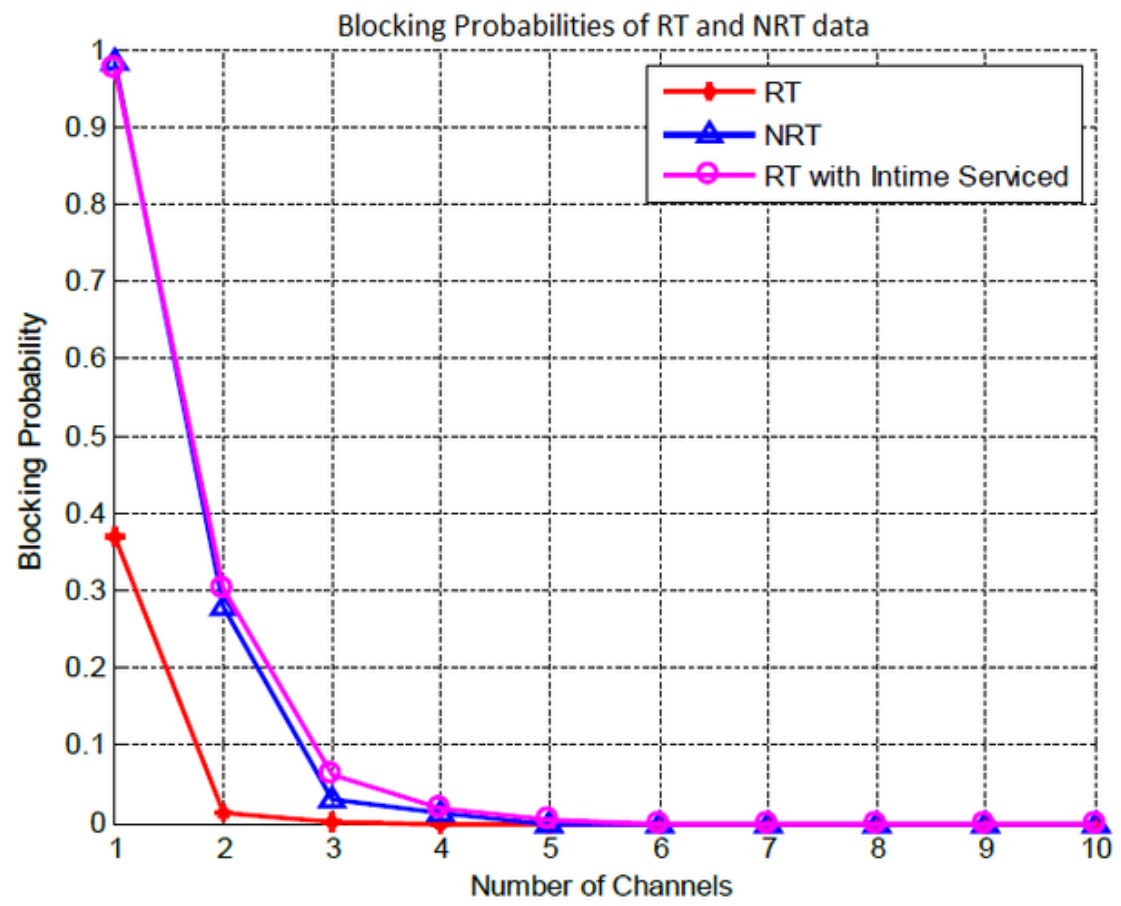

Figure 6. Blocking Probability of Secondary users with Queue Shifting, for High PU Occupancy and Low SU Demand

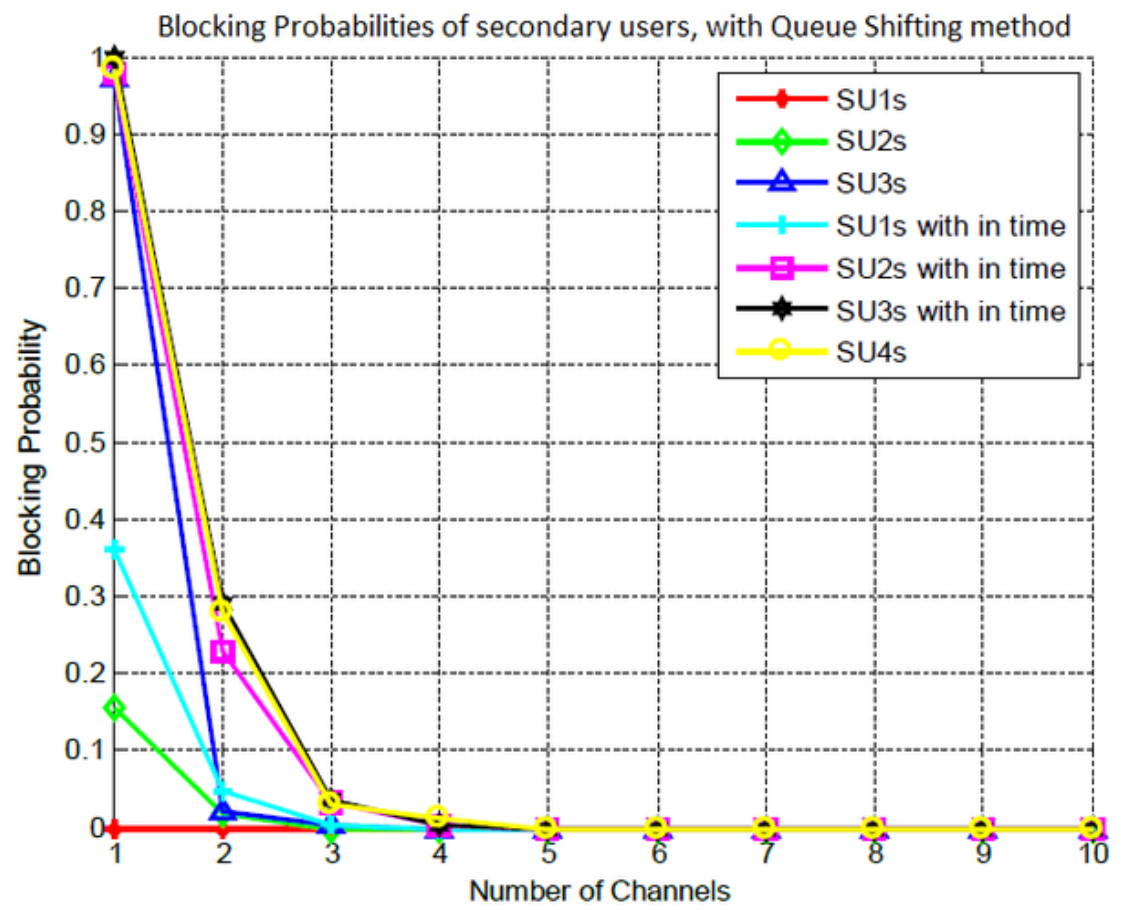


Figure 7. Blocking Probability of Real-Time SUs with and without Queue Shifting, for High PU Occupancy and Low SU Demand

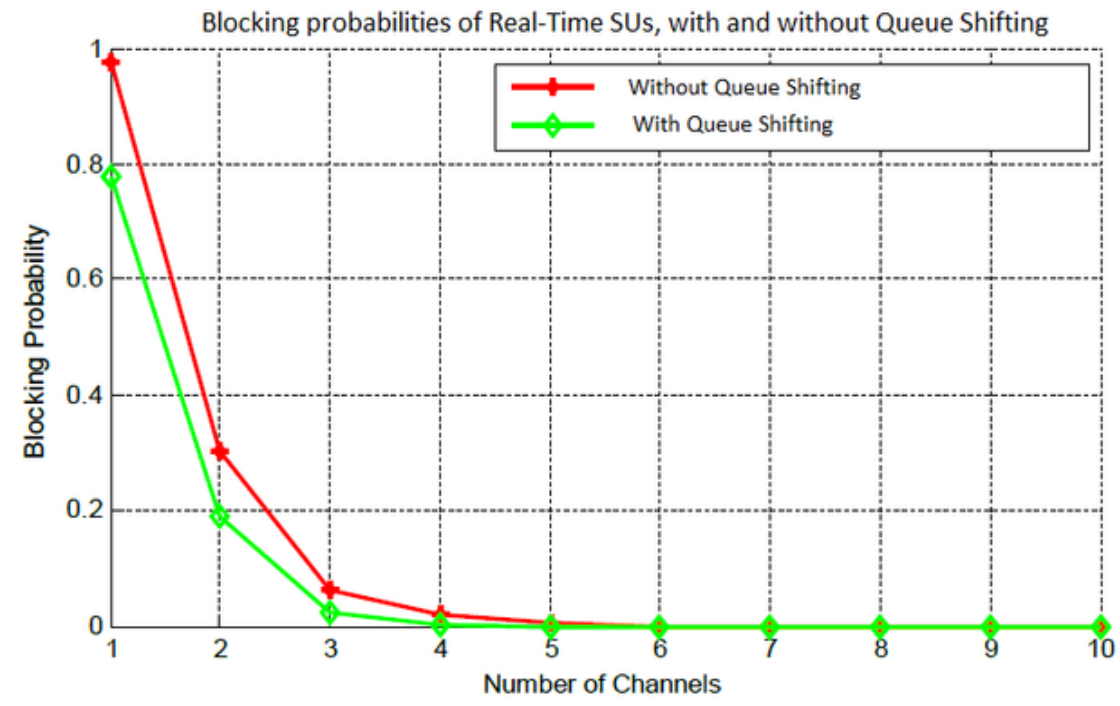

Figure 8. Blocking Probability of RT and NRT Secondary Users for High PU Occupancy and High SU Demand

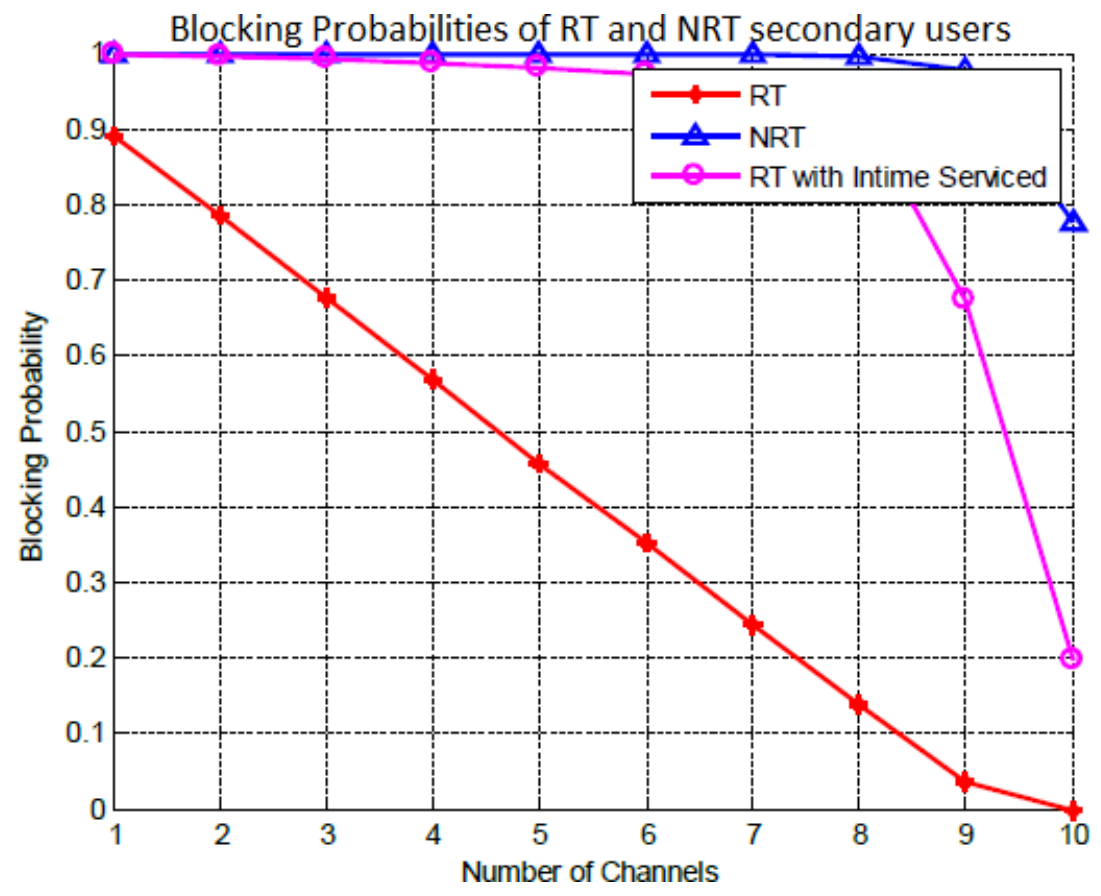

between Figure 8 and 10 is that, in Figure 10, summation of SU1 through SU3 is considered for RT type of packets, whereas in Fig-8, there are only two types of packets namely, RT and NRT. For this case of high SU demand, improvements are found to be in the range of $10 \%$ to $50 \%$, depending on the number of vacant channels indicated on $\mathrm{x}$-axis. 
Figure 9. Blocking Probability of Secondary users with Queue Shifting, for High PU Occupancy and High SU Demand

Blocking probabilities of șecondary users with Queue Shifting method

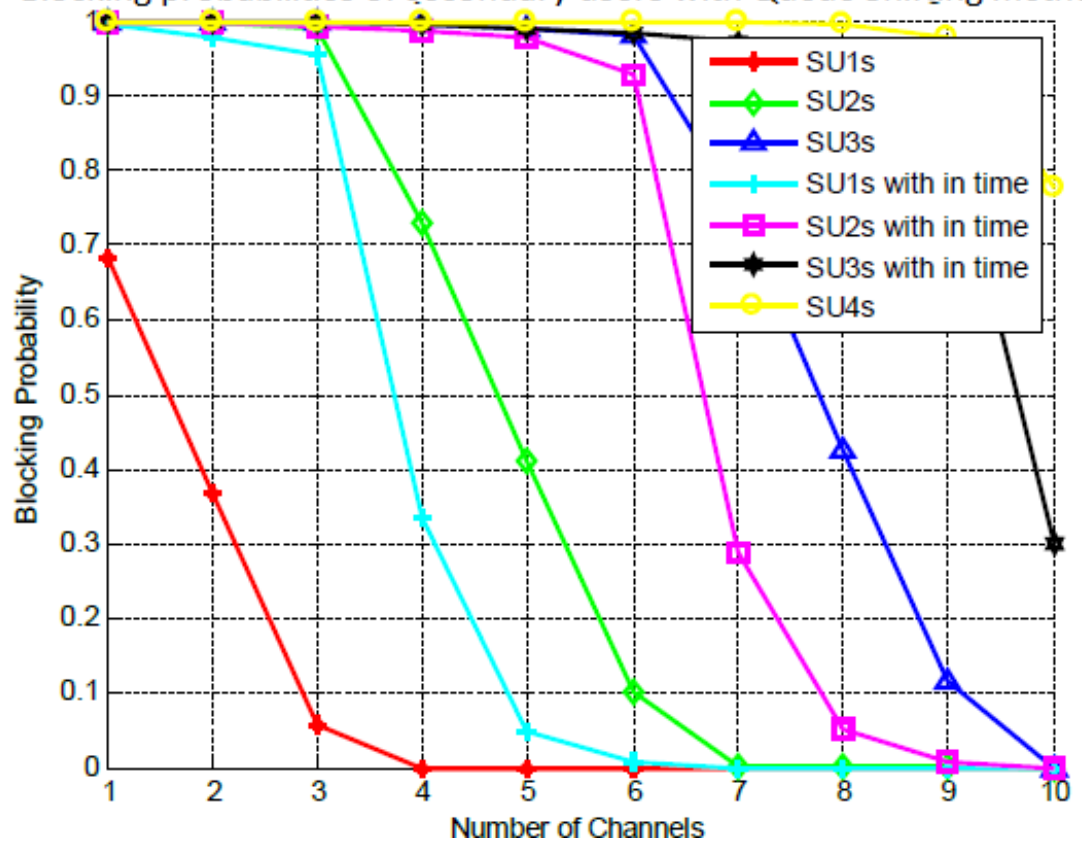

Figure 10. Blocking Probability of Real-Time SUs with and without Queue Shifting, for High PU Occupancy and High SU Demand

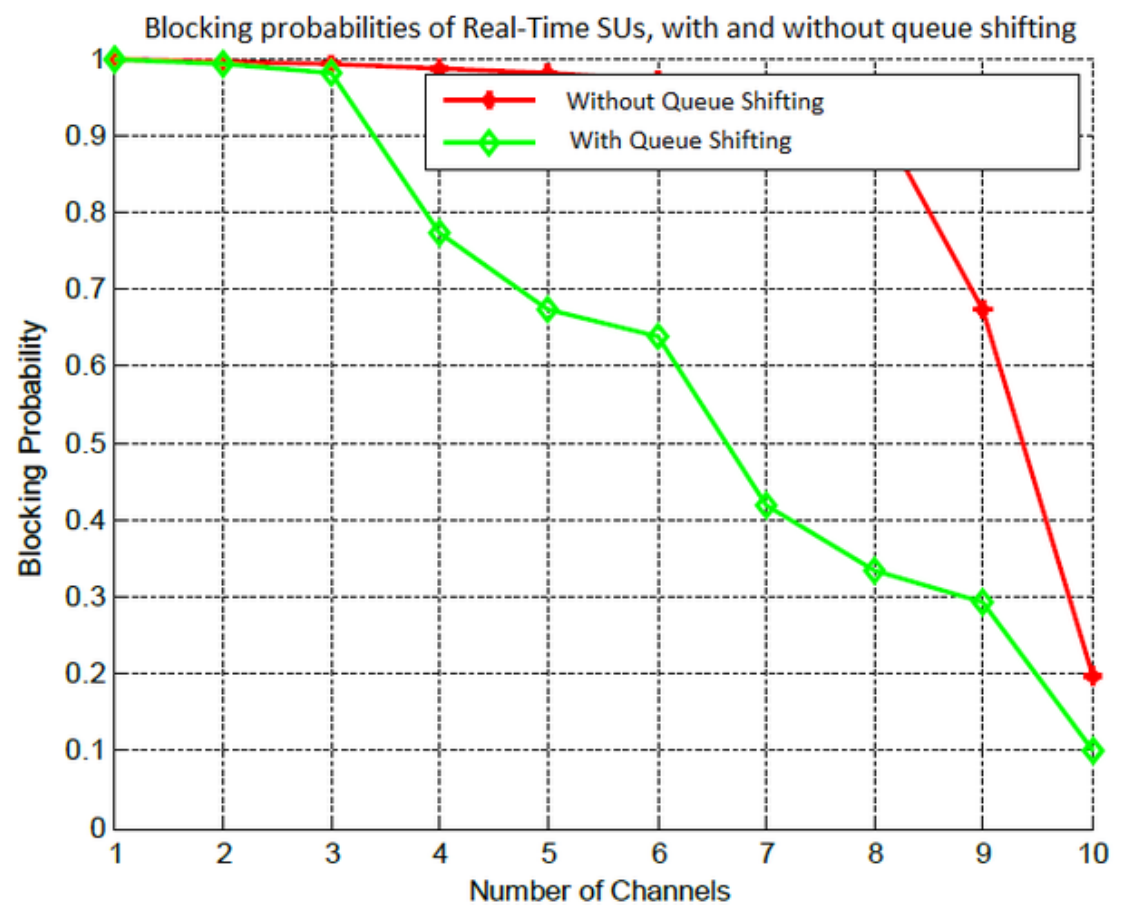


When the SU demand is more, the number of requests from the users of all priorities increase. In such situations, the system will help in bringing quickly the packets of high priority users to the queue that is connected to transmission line. The advantage of multiple queues is that the packets that cannot tolerate higher delays will quickly move to the upper queues, when compared to the methods of RT-NRT based treatment of packets. Due to the proposed multiple queues and queue shifting mechanism, the less real-time packets will give way to the more real-time packets, even if the former type have entered the system queue early. The less real-time and more real-time here refer to the packets of lesser time-sensitive and higher time-sensitive nature of the data, even though both of them are real-time packets in the broader sense.

\section{CONCLUSION AND FUTURE SCOPE}

A mechanism that is based on queue-shifting is proposed for spectrum sharing in cognitive radio networks. It is meant for offering prioritized channel allocations to real-time data packets of users. Making the packets of different 'Tolerable Intervals' to enter the respective queues, and escalate them to the upper-level queues as time elapses, is the main aspect of it. Finally, the packets that enter the top-level first queue will be transmitted, one after the other, in the order in which they entered this queue. Markov-chain based models for various scenarios are considered for analysis. Simulation studies are carried out through discrete event simulation scenarios generated through Matlab. The scenarios of LOW and HIGH demand from secondary users in the context of HIGH occupancy by primary users are considered. Reduced blocking probabilities of real-time data are observed for these scenarios. Improvements of upto $20 \%$ are observed for the LOW demand scenarios and improvements of $10 \%-50 \%$ are observed for HIGH demand scenario. One user of each priority is considered in this simulation. The case of multiple users in each category is considered as equivalent to higher demand from that one user, in this work. However, considering multiple users in each category with their arrival rates at different instants can be carried out for better insights into it. Also, simulating the models with the appropriate communication elements that include the practical details like bit errors, transmission delays etc., may be carried out for further understanding of it. 


\section{REFERENCES}

Akyildiz, I. F., Lee, W. Y., Vuran, M. C. \&Mohanty, S. (2008). A survey on spectrum management in cognitive radio networks. IEEE Communications Magazine, 46(4), 40-48.

Alshamrani, A., Shen, X. S., \& Xie, L. L. (2011). QoS Provisioning for Heterogeneous Services in Cooperative Cognitive Radio Networks. IEEE Journal on Selected Areas in Communications, 29(4), 819-830. doi:10.1109/ JSAC.2011.110413

Annapurna, K., \& Ramanjaneyulu, B. S. (2019). QoS maintenance in cognitive radio networks with prioritysupporting novel channel allocation method. International Journal of Business Data Communications and Networking, 15(1), 1-16. doi:10.4018/IJBDCN.2019010101

Balapuwaduge, I. A. M., Jiao, L., Pla, V., \& Li, F. Y. (2014). Channel Assembling with Priority-Based Queues in Cognitive Radio Networks: Strategies and Performance Evaluation. IEEE Transactions on Wireless Communications, 13(2), 630-645. doi:10.1109/TWC.2013.120713.121948

Wang, Wu, \& Ray Liu. (2010). Game Theory for Cognitive Radio Networks: An overview. Computer Networks, 54(14), 2537-2561.

El Helou, M., Ibrahim, M., Lahoud, S., Khawam, K., Mezher, D., \& Cousin, B. (2015). A Network Assisted Approach for RAT selection in Heterogeneous Cellular Networks. IEEE Journal on Selected Areas in Communications, 33(6), 1055-1067. doi:10.1109/JSAC.2015.2416987

Tragos, E. Z., Zeadally, S., Fragkiadakis, A. G., \& Siris, V. A. (2013). Spectrum Assignment in Cognitive Radio Networks: A Comprehensive Survey. IEEE Communications Surveys and Tutorials, 15(3), 2013. doi:10.1109/ SURV.2012.121112.00047

Feng, S., \& Zhao, D. (2010). Supporting Real-Time CBR Traffic in a Cognitive Radio Sensor Network. IEEE Wireless Communication and Networking Conference, 1-6. doi:10.1109/WCNC.2010.5506276

Goldsmith, A., Jafar, S. A., Maric, I., \& Srinivasa, S. (2009). Breaking spectrum gridlock with cognitive radios: An information theoretic perspective. Proceedings of the IEEE, 97(5), 894-914. doi:10.1109/JPROC.2009.2015717

Homayounzadeh, A., \& Mahdavi, M. (2017). Quality of Service Analysis for the Real-Time Secondary Users in Cognitive Radio Cellular Networks. Wireless Personal Communications, 96(2), 3041-3061. doi:10.1007/ s11277-017-4340-y

IEEE Std 802.11 (2016), IEEE Standard for Information technology-Telecommunications and information exchange between systems Local and metropolitan area networks-Specific requirements - Part 11: Wireless LAN Medium Access Control (MAC) and Physical Layer (PHY) Specifications, IEEE Std 802.11-2016 (Revision of IEEE Std 802.11-2012), vol., no., pp.1-3534, 14 Dec. 2016

Jayaweera, S. K., Vazquez-Vilar, G., \& Mosquera, C. (2010). Dynamic Spectrum Leasing: A New Paradigm for Spectrum Sharing in Cognitive Radio Networks. IEEE Transactions on Vehicular Technology, 59(5), 2328-2339. doi:10.1109/TVT.2010.2042741

Kunert, K., Jonsson, M., \& Bilstrup, U. (2012). Deterministic real-time medium access for cognitive industrial radio networks. Proceedings of 9th IEEE International Workshop on Factory Communication Systems, 91-94. doi:10.1109/WFCS.2012.6242549

Liang, Z., Feng, S., Zhao, D., \& Shen, X. S. (2011). Delay Performance Analysis for Supporting Real-Time Traffic in a Cognitive Radio Sensor Network. IEEE Transactions on Wireless Communications, 10(1), 325-335. doi:10.1109/TWC.2010.111910.100804

Mitola, J. (2009). Cognitive Radio Architecture Evolution. Proceedings of the IEEE, 97(4), 626-641. doi:10.1109/ JPROC.2009.2013012

Motamedi, N., Kumar, S., Hu, F., \& Rowe, N. (2013). A priority-aware channel selection scheme for real-time data transmission in cognitive radio networks. 2013 International Conference on Computing, Networking and Communications (ICNC), 734-739 doi:10.1109/ICCNC.2013.6504179

Niyato, D., \& Hossain, E. (2007). A game theoretic approach to competitive spectrum sharing in cognitive radio networks. IEEE Wireless Communications and Networking Conference, 16-20. doi:10.1109/WCNC.2007.9 
Smith, Firag, Dmochowski, \& Shafi. (2012). Analysis of the M/M/N/N Queue with Two Types of Arrival Process: Applications to Future Mobile Radio Systems. Journal of Applied Mathematics, 1-14.

Shiang, H., \& Van der Schaar, M. (2008). Queuing-Based Dynamic Channel Selection for Heterogeneous Multimedia Applications Over Cognitive Radio Networks. IEEE Transactions on Multimedia, 10(5), 896-909. doi:10.1109/TMM.2008.922851

Suganthi, N., \& Meenakshi, S. (2018). An efficient scheduling algorithm using queuing system to minimize starvation of non-real-time secondary users in Cognitive Radio Network. Cluster Computing. Advance online publication. doi:10.1007/s10586-017-1595-8

Wang, F., Huang, J., \& Zhao, Y. (2012). Delay Sensitive Communications over Cognitive Radio Networks. IEEE Transactions on Wireless Communications, 11(4), 1402-1411. doi:10.1109/TWC.2012.020812.110133

Won-Yeol Lee, , \& Akyildiz, I. F. (2008). Optimal spectrum sensing framework for cognitive radio networks. IEEE Transactions on Wireless Communications, 7(10), 3845-3857. doi:10.1109/T-WC.2008.070391

Yang, W., \& Zu, Y. (2016). The Research of QoS Guarantee Mechanism of the Secondary User in Cognitive Radio Networks, Communication Networks. China Communications, 13(10), 146-152. doi:10.1109/CC.2016.7733039

Choi \& Shin. (2011). Opportunistic Access of TV Spectrum Using Cognitive-Radio-Enabled Cellular Networks. IEEE Transactions on Digital Object Identifier, 60(8), 3853 - 3864.

Yu, P. X., Tan, X., Yu, T. L., Ma, L., \& Hai, Y. W. (2011). Spectrum allocation algorithm based on game theory, 2011 international conference on Instrumentation. Measurement, Computer, Communication and Control.

Babburi Seetha Ramanjaneyulu received his Bachelor of Engineering degree in Electronics and Communication Engineering from Andhra University, India. He completed his Master of Engineering program from Bangalore University in Electronics discipline and Ph.D., from National Institute of Technology, Calicut, India in 2007, in Wireless Networks domain. He has worked as Design Engineer and Senior Design Engineer in NIELIT, and as Member Technical Staff in CDAC. He is working as Professor in VFSTR University Guntur, from the year 2008. His research interests are in Computer Networks, Wireless Communications, Wireless Sensor Networks, Cognitive Radio Networks, Cellular Communication Systems, Femtocells, Interference Mitigation in MANETs, and Real-Time data transmissions on Wire and Wireless Networks.

K. Annapurna was born in Andhra Pradesh, India in 1979. She obtained her diploma in Electronics and Communication Engineering in 1997 from State Board of Technical Education and Training, and B. Tech in Electronics and Communications Engineering in 2001 from Acharya Nagarjuna University. She completed her M. Tech in Digital Systems and Computer Electronics in 2011 from Jawaharlal Nehru Technological University and Ph.D in Cognitive Radio Networks in 2019 from VFSTR University. Her research interests include Cognitive Radio Networks, Non Orthogonal Multiple Access for $5 \mathrm{G}$ and Optimization algorithms. She has published 25 research papers in peer-reviewed journals and conferences. She is a member of IEEE and reviewer for International Journal of Wireless Networks and Broadband Technologies. She has a total teaching and research experience of 18 years. 